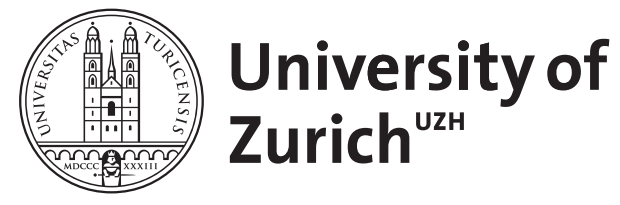

\title{
Re: Rivaroxaban for Thromboprophylaxis in High-risk Ambulatory Patients
} with Cancer

\author{
Fankhauser, Christian Daniel ; Sweeney, Christopher J ; Connors, Jean M
}

DOI: https://doi.org/10.1016/j.eururo.2019.10.019

Posted at the Zurich Open Repository and Archive, University of Zurich ZORA URL: https://doi.org/10.5167/uzh-176989

Journal Article

Accepted Version

Originally published at:

Fankhauser, Christian Daniel; Sweeney, Christopher J; Connors, Jean M (2020). Re: Rivaroxaban for Thromboprophylaxis in High-risk Ambulatory Patients with Cancer. European Urology, 77(3):388-390. DOI: https://doi.org/10.1016/j.eururo.2019.10.019 


\section{Which genitourinary cancer patient should get}

\section{prophylactic anticoagulation?}

Christian Daniel Fankhauser ${ }^{1}$, Christopher J Sweeney ${ }^{2}$ Jean M. Connors ${ }^{3}$

${ }^{1}$ Department of Urology, University of Zurich, Zurich, Switzerland

${ }^{2}$ Hematology Division, Brigham and Women's Hospital, Harvard Medical School, Boston, MA, USA

${ }^{3}$ Dana-Farber Cancer Institute, Harvard Medical School, Boston, MA, USA

Khorana AA, Soff GA, Kakkar AK, Vadhan-Raj S, Riess H, Wun T, et al. Rivaroxaban for Thromboprophylaxis in HighRisk Ambulatory Patients with Cancer. New England Journal of Medicine. 2019

Carrier M, Abou-Nassar K, Mallick R, Tagalakis V, Shivakumar S, Schattner A, et al. Apixaban to Prevent Venous Thromboembolism in Patients with Cancer. The New England journal of medicine. 2019

\section{Experts' summary}

Venous thromboembolic events (VTE) are common complications in patients with cancer [1]. In the CASSINI and AVERT trials ambulatory patients receiving systemic cancer therapy with an intermediate-to-high risk for VTE (Khorana score, $\geq 2$ ) were randomized to either placebo or direct oral anticoagulants (DOACs) [2, 3]. The hazard ratios (HR) for VTE were reported as 0.66 and 0.41 together with HRs for bleeding of 1.96 and 2.00 respectively.

\section{Experts' comments}

The CASSINI and AVERT trials showed that prophylactic use of DOACs in cancer patients undergoing systemic therapy with an increased risk for VTE halves the risk of VTE and doubles the risk of bleeding [4, 5]. Previous findings for low molecular weight heparins $(\mathrm{LMWH})$ found a similar relative risk reduction but a lower absolute risk reduction because of the less selective inclusion of patients. Ideally, our decisions would be based on randomized controlled trials powered to analyze the effect and risk of VTE in each genitourinary cancer individually, but unfortunately no such trial can be expected in the 
near future and physicians have to decide upon VTE prophylaxis on a daily base. In this report, we (1) underline the challenges to apply the current literature in genitourinary cancer patients, (2) provide a simple tool to decide which patient should get prophylactic anticoagulation and (3) draw attention to an important research niche.

The main challenge is to identify patients at a sufficiently high risk for VTE to justify prophylactic anticoagulation, as there is an associated increased risk of bleeding. The CASSINI and AVERT trials used the Khorana risk model to determine a patient's VTE risk. This model including five risk factors, was developed with 2701 patients but had a low rate of only 60 events. It was then validated in a cohort of 1365 patients that experienced even fewer events [6]. The model cohort included primarily adenocarcinoma, sarcoma and lymphoma patients, which each have distinct VTE etiologies - some of which are innate to the histology and others to local compression of venous system. Genitourinary cancers, including various histologies such as urothelial, clear cell, adenocarcinomas and germ cell tumors were not well represented in either the derivation or validation cohorts. Therefore, the Khorana risk model may not be adequate to determine the risk of VTE in genitourinary cancer patients and better genitourinary cancer specific risk scores are needed. How to determine the threshold for using VTE prophylaxis is currently unclear.

The cut-off to initiate prophylaxis can be derived from CASSINI and AVERT trials as follows: The HR can be used to estimate the number needed to treat (NNT) with the following formula [7]:

$$
N N T=\frac{1}{(1-\text { cumulative incidence })^{\mathrm{HR}_{-}}(1-\text { cumulative incidence })}
$$

As shown in this formula, the NNT is not only influenced by the HR but also substantially driven by the cumulative VTE incidence. We plotted all estimated NNT for HRs between 0.4-0.7 for a very low (1\%) up to a very high (30\%) cumulative VTE incidence in patients 
without prophylaxis. The NNT versus VTE risk curve illustrates that patients with a low risk of VTE do not benefit from prophylaxis. However, as the NNT decreases rapidly even with a more conservative HR of 0.7 for efficacy, the NNT falls below the number needed to harm (NNH) at a cumulative VTE incidence around 6\%. In addition to calculating the NNT and NNH, other factors influence the use of anticoagulants in the prophylactic setting including ease of administration and financial cost to the patient. At a more general level, health economic modeling is needed to determine benefits to the society and this in turn is predicated by payer models. This would also account for the cost of care for major pulmonary embolism requiring inpatient care, long term care of venous insufficiency and rare but lethal complications of bleeding and pulmonary embolism.

This simple decision aid supports the treating clinician when trying to decide whether a patient should receive prophylactic anticoagulation or not. For example, prostate cancer patients during first- or second-line chemo- or hormonal therapy have a negligible cumulative VTE incidence and therefore the NNT would be unreasonably high in most cases. Another patient population at high risk for bleeding, such as patients with untreated primary in the urinary tract, would have a substantially lower $\mathrm{NNH}$ (red line in plot), changing the risk-benefit ratio. We previously reported cumulative incidence VTE risks of over $>8 \%$ in metastatic germ cell cancer patients [8], indicating that the NNT may be lower than the $\mathrm{NNH}$ and therefore prophylaxis could be justified. However, these cumulative VTE incident rates have to be confirmed in patients with or without other VTE risk factors. In summary, we acknowledge that the presented approach includes several strong assumptions, which need to be proven; therefore there is a need to develop genitourinary cancer specific VTE and bleeding risk scores to facilitate decision-making. In the meantime, this commentary can help clinicians taking care of patients with genitourinary malignancies decide on when to use prophylactic anti-coagulation. 


\section{Figure legend}

Figure 1 NNT versus VTE risk curve Comparing the modeled number needed to treat according to cumulative risk of having a venous thrombo-embolic event against the number needed to harm.

\section{References}

[1] Connors JM. Prophylaxis against Venous Thromboembolism in Ambulatory Patients with Cancer. New England Journal of Medicine. 2014;370:2515-9.

[2] Khorana AA, Soff GA, Kakkar AK, Vadhan-Raj S, Riess H, Wun T, et al. Rivaroxaban for Thromboprophylaxis in High-Risk Ambulatory Patients with Cancer. New England Journal of Medicine. 2019;380:720-8.

[3] Carrier M, Abou-Nassar K, Mallick R, Tagalakis V, Shivakumar S, Schattner A, et al. Apixaban to Prevent Venous Thromboembolism in Patients with Cancer. The New England journal of medicine. 2019;380:7119.

[4] Agnelli G, George DJ, Kakkar AK, Fisher W, Lassen MR, Mismetti P, et al. Semuloparin for thromboprophylaxis in patients receiving chemotherapy for cancer. The New England journal of medicine. 2012;366:601-9.

[5] Agnelli G, Gussoni G, Bianchini C, Verso M, Mandala M, Cavanna L, et al. Nadroparin for the prevention of thromboembolic events in ambulatory patients with metastatic or locally advanced solid cancer receiving chemotherapy: a randomised, placebo-controlled, double-blind study. The Lancet Oncology. 2009;10:943-9.

[6] Khorana AA, Kuderer NM, Culakova E, Lyman GH, Francis CW. Development and validation of a predictive model for chemotherapy-associated thrombosis. Blood. 2008;111:4902-7.

[7] Altman DG, Andersen PK. Calculating the number needed to treat for trials where the outcome is time to an event. BMJ. 1999;319:1492-5.

[8] Bezan A, Posch F, Ploner F, Bauernhofer T, Pichler M, Szkandera J, et al. Risk stratification for venous thromboembolism in patients with testicular germ cell tumors. PloS one. 2017;12:e0176283.

\section{Acknowledgments}

CD Fankhauser is supported by funding from the University Hospital of Zurich and medAlumni Zurich, Stiftung für urologische Forschung, Marlis Geiser-Lemken Stiftung, Fonds zur Förderung des akademischen Nachwuchses (FAN), Ernst Göhner Stiftung, SAKK/Dr. Paul Janssen Fellowship, Arnold U. und Susanne Huggenberger-Bischoff Stiftung zur Krebsforschung 